\title{
Late gadolinium enhancement by cardiovascular magnetic resonance provides prognostic information in symptomatic alcoholic cardiomyopathy
}

Wei Xiangli

From 18th Annual SCMR Scientific Sessions

Nice, France. 4-7 February 2015

\section{Background}

Previous studies have demonstrated that late gadolinium enhancement (LGE) on cardiovascular magnetic resonance (CMR)predicted independently cardiac adverse outcomes in dilated cardiomyopathy (DCM). ACM patients did not have a better outcome than DCM and prognosis was poorer after development of heart failure. There is no report about the association between alcoholic cardiomyopathy (ACM) outcomes and LGE.

\section{Methods}

70 consecutive ACM patients from July 2008 to December 2010 were examined with LGE during CMR. The mean follow-up time was $42.3 \pm 17.5$ months after CMR. Cardiac events include cardiac death, implantable cardioverter-defibrillator (ICD) discharge, hospitalisation for decompensated congestive heart failure (CHF) and heart transplantation.

\section{Results}

Of the 70 patients, myocardial fibrosis visualized by LGE was detected in 24(34.3\%) patients. During the follow-up period, most events $(n=9)$ were related to hospitalisation for decompensated CHF in the total 13 cardiac events. The incidence of cardiac events was significantly higher in patients with LGE than that without LGE (37.5\% vs. 8.7\%, $\mathrm{P}=0.003)$. When entered into multivariate Cox regression analysis, the presence of LGE yields hazard ratio (HR) of 4.62 (95\% CI, 1.4 to 15.4$)$ for cardiac events $(\mathrm{P}=0.007)$, the extent of LGE also retains its independent predictive value in LGE (+) patients with HR of 1.13 (95\% CI, 1.04 to 1.22, $\mathrm{P}=0.003)$.

\section{Conclusions}

In patients with symptomatic ACM, the presence of LGE determined by CMR is a strong independent predictor of adverse cardiac events.

\section{Funding}

This study was supported by grant No. 81130029 from the key projects of National Natural Science Foundation of China and by grant (Z121107001012114) from Peking Science and Technology Commission.

Published: 3 February 2015

doi:10.1186/1532-429X-17-S1-P316

Cite this article as: Xiangli: Late gadolinium enhancement by

cardiovascular magnetic resonance provides prognostic information in

symptomatic alcoholic cardiomyopathy. Journal of Cardiovascular Magnetic Resonance 2015 17(Suppl 1):P316. 